\title{
A Simple and Versatile Reactor for Photochemistry
}

Charlotte A. Clark, ${ }^{a}$ Darren S. Lee, ${ }^{\text {a }}$ Stephen J. Pickering, ${ }^{b}$ Martyn Poliakoff*a and Michael W. George*a, c

${ }^{a}$ School of Chemistry, The University of Nottingham, University Park, Nottingham, NG7 2RD, UK.

${ }^{b}$ Department of Mechanical, Materials and Manufacturing Engineering, The University of Nottingham, University Park, Nottingham NG7 2RD, UK.

${ }^{c}$ Department of Chemical and Environmental Engineering, The University of Nottingham Ningbo China, 199 Taikang East Road, Ningbo 315100, China.

Safety warning: Any experiment involving flammable organic solvents and air or pure oxygen is potentially hazardous, especially when partially contained, as is the case of the flask of our reactor. We took the following precautions and encountered no problems but we stress the need for readers to make safety assessments for their own experiments as peripheral circumstances may be different from ours. All experiments were carried out in a fume hood or ventilated enclosure with adequate ventilation and the front lowered. Any obvious sources of ignition were removed. Oxygen was fed from a cylinder fitted with a compliant regulator and was delivered at a maintained pressure of 1 bar using a mass flow controller compatible with oxygen. The equipment was maintained and cleaned free of grease at all times to prevent any incompatibilities with oxygen. Temperatures were kept at ambient. When working above the solvent flash point and LOC, care must be taken to ensure that all possible risks have been considered. Appropriate safeguards and suitable safety measures must be implemented. 


\section{Electronic Supporting Information}

\section{Experimental}

All chemicals were purchased from standard suppliers and used as received without further purification. Quantitative ${ }^{1} \mathrm{H}$ NMR yields were determined using an internal standard of 0.1 equivalents of biphenyl.

NMR spectra were obtained using a Bruker DPX300 spectrometer.

\section{Reactor Setup}

The photoreactor is based around an IKA RV10-Control rotary evaporator fitted with $50 \mathrm{~mL}$, $1 \mathrm{~L}, 2 \mathrm{~L}$ or $3 \mathrm{~L}$ flasks - for the $1 \mathrm{~L}$ flask both round-bottomed (RB) and pear-shaped (PS) flasks were used with similar results obtained indicating minimal differences between the two flask shapes. The pumps used are Masterflex peristaltic pumps with PTFE tubing used throughout. Oxygen was dosed into the reaction flask using a Bronkhorst EL-Flow mass-flow controller set at a fixed rate of $60 \mathrm{sccm}$ at a pressure of $1.015 \mathrm{bar}$.

Automated PC control of the reactor was achieved via the construction of a control board, built at Nottingham, which utilises an Arduino Uno microcontroller. All commands to the microcontroller were written and uploaded using Arduino 1.6.5.

FTIR spectra were recorded using a Bruker Alpha ATR with a flow accessory enabling incorporation of the spectrometer into the flow system of reactor between the reactor and the product collection flask. Spectra were recorded at a resolution of $4 \mathrm{~cm}^{-1}$ with 32 scans.

The LEDs used in our initial experiments were two fan cooled banks (each containing 5 units) of OSRAM OSTAR 1000 lumen LEDs (Part No: LE CW E3B. In the higher light intensity experiments, two banks (each composed of three units) of Citizen CLL042-1818A550K1A1 8000 Lumen LEDs were used. The LED assemblies were mounted onto custom water cooled blocks, with a Grant LTD6G bath used to maintain the temperature of the block below $30^{\circ} \mathrm{C}$ with closed-cycle cooling. Although, this was not undertaken, mains water could have been used instead to provide the required cooling. The setup of the LED blocks were positioned on the right hand side of the water bath, angled to face the reaction flask and placed in close proximity (within $c a .2 \mathrm{~cm}$ ) to the reaction flask, shown in Figure ES4. This positioning of the LEDs was chosen to irradiate the bottom of the reaction flask, shown by 
our film measurements to be the location of the thinnest film for more efficient irradiation. The LEDs were kept in fixed positions throughout experiments.

\section{Experimental procedures}

Preparation of all reaction solutions was undertaken in amber-coloured glassware and shielded from light prior to photolysis. The determined residence time for each reaction are taken from residence time studies. In these experiments a fixed volume of solution was irradiated for a specific residence time. For the next residence time a new aliquot of reaction solution was used, enabling testing of the reproducibility of the reactions studied. 


\section{Photo de-symmetrisation of para-diphenyboronic acid, (1).}

For these experiments a stock solution of $0.05 \mathrm{M} p$-benzene diboronic acid was used with 2 mol- $\%$ rose bengal as the photosensitizer with 2 molar equiv. of DIPEA. Aliquots were taken for conversion monitoring and the solution was immediately quenched with $1 \mathrm{M} \mathrm{HCl}$ before the solvent evaporated. ${ }^{1} \mathrm{H}$ NMR was used to determine the reaction conversions. NMR data collected are in accordance with that found in the literature.

${ }^{1} \mathrm{H}$ NMR for 2 (DMSO-d $6,300 \mathrm{MHz}$ ): $\delta$ 9.27, (br, s, $\left.1 \mathrm{H}, \mathrm{Ar}-\mathrm{OH}\right), 7.59$ (br d, $J=8.4 \mathrm{~Hz}, 2 \mathrm{H}$, Ar-H), 6.70 (br d, $J=8.4 \mathrm{~Hz}, 2 \mathrm{H}, \mathrm{Ar}-\mathrm{H}$ ). ${ }^{1} \mathrm{H}$ NMR for 3 (DMSO-d $6,300 \mathrm{MHz}, \mathrm{ppm}$ ): $\delta 8.68$ (br, s, 1H Ar-OH), 6.55 (s, 4H, Ar-H).

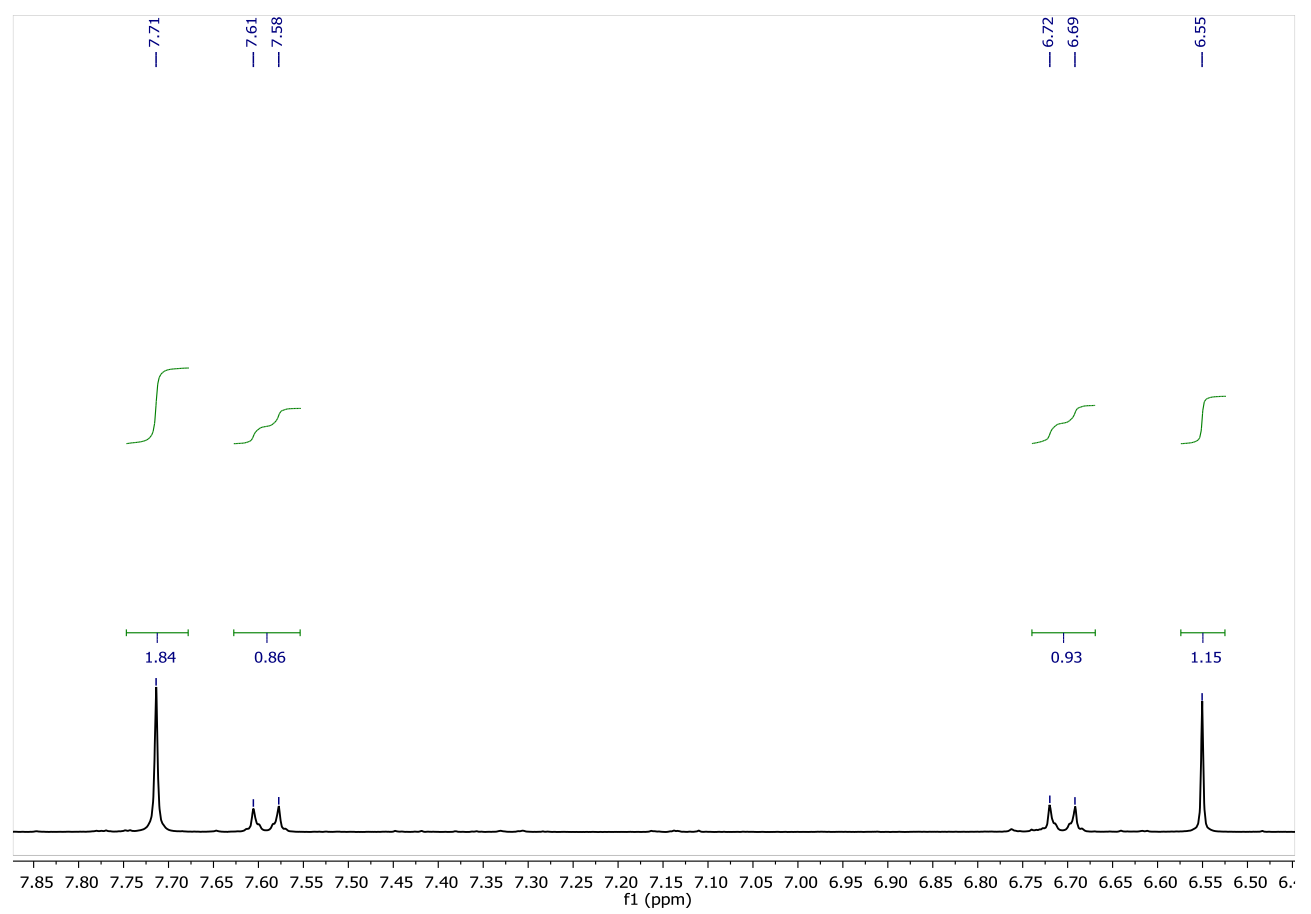

Figure ES1 - Expanded ${ }^{1}$ H NMR spectrum recorded after 5 mins photolysis of 1 showing the formation of both photoproducts $\mathbf{2}$ and $\mathbf{3}$. 


\section{Photo-oxidation of $\alpha$-terpinene, (4).}

All experiments detailed were conducted using a fixed concentration of $0.1 \mathrm{M} \alpha$-terpinene in EtOH with 1 mol-\% rose bengal. For the reactions conducted in solvents other than EtOH, 1mol\% tetraphenylporphyrin (TPP) was used as the photosensitizer. Following the reaction, aliquots were taken for ${ }^{1} \mathrm{H}$ NMR and the solvent evaporated. Quantitative ${ }^{1} \mathrm{H}$ NMR was utilised to determine the yield, using biphenyl ( 0.1 equivalents) as an internal standard and using signals between 7.60-6.30 ppm as shown below. In all experiments only small amounts of the side-product $p$-cymene were apparent (s at $7.11 \mathrm{ppm}, 4 \mathrm{H}$ ). Typical max. yield for this reaction was $c a .78 \%$. NMR data are in agreement with literature values, with only small amounts of the known side product $p$-cymene present by ${ }^{1} \mathrm{H}$ NMR. The $\alpha$-terpinene used (FG, $\geq 89 \%$ ) showed only small amounts of $p$-cymene and no detectable ascaridole present by ${ }^{1} \mathrm{H}$ NMR.

${ }^{1} \mathrm{H}$ NMR for $5\left(\mathrm{CDCl}_{3}, 300 \mathrm{MHz}\right) \delta 6.50(\mathrm{~d}, J=8.5 \mathrm{~Hz}, 1 \mathrm{H}, \mathrm{CH}), 6.41(\mathrm{~d}, J=8.5 \mathrm{~Hz}, 1 \mathrm{H}$, $\mathrm{CH})$.

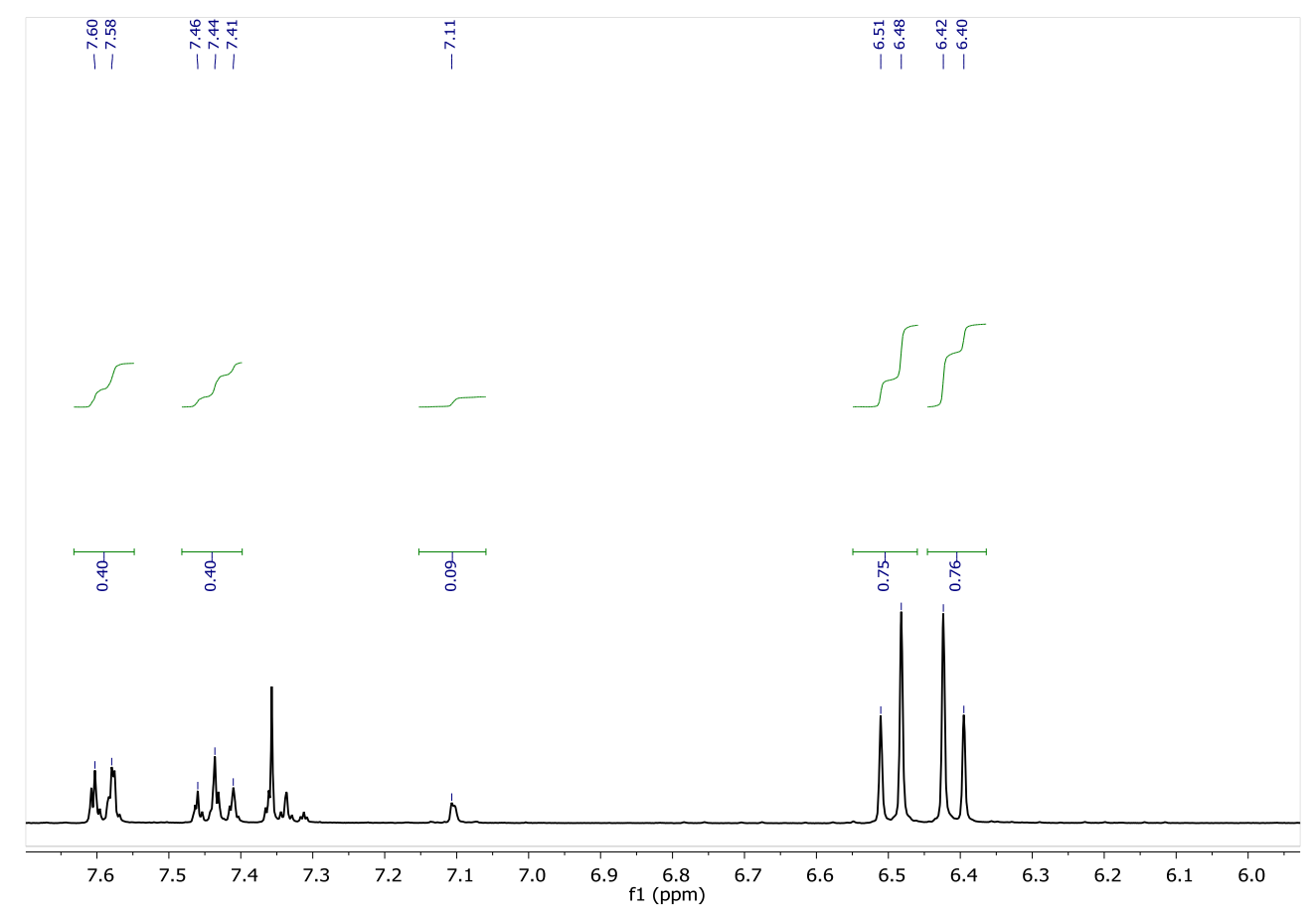

Figure ES2 - Expanded ${ }^{1} \mathrm{H}$ NMR spectrum recorded after 30 s photolysis of 4 showing the formation of 5 (Table 1, entry 2). p-cymene (7.11 ppm) and biphenyl as the internal standard (7.3-7.6 ppm). 


\section{Photo-oxidation of citronellol, (6).}

For each reaction, the appropriate quantity of citronellol was added to EtOH to make up the required concentration $(0.1-1 \mathrm{M})$. In $\mathrm{EtOH}$, rose bengal was used as the photosensitizer, typically at a concentration of $3 \mathrm{mM}$ (reduced to $1 \mathrm{mM}$ at lower conc. sensitizer experiments). For reactions in $\mathrm{CH}_{2} \mathrm{Cl}_{2}, 2$ mol-\% TPP was used as a photosensitizer. Following photolysis, the ATR-FTIR spectra of the hydroperoxides ( $7 \& 8$ ) were measured and the solution collected. The hydroperoxides were quenched using excess triphenylphosphine in $\mathrm{Et}_{2} \mathrm{O}$ and the solvent was evaporated. The conversions to the diols $(9$ \& 10) were determined by ${ }^{1} \mathrm{H}$ NMR using signals in the $4.70-5.70 \mathrm{ppm}$ region as indicated below. The average ratio of 9:10 was determined to be 1.15:1 from ${ }^{1} \mathrm{H}$ NMR. NMR data are in agreement with literature values.

${ }^{1} \mathrm{H}$ NMR for $9\left(\mathrm{CDCl}_{3}, 300 \mathrm{MHz}\right) \delta 5.72-5.60(\mathrm{~m}, 2 \mathrm{H})$.

${ }^{1} \mathrm{H}$ NMR for $10\left(\mathrm{CDCl}_{3}, 300 \mathrm{MHz}\right) \delta 4.98(\mathrm{~m}, 1 \mathrm{H}), 4.87(\mathrm{~m}, 1 \mathrm{H})$.

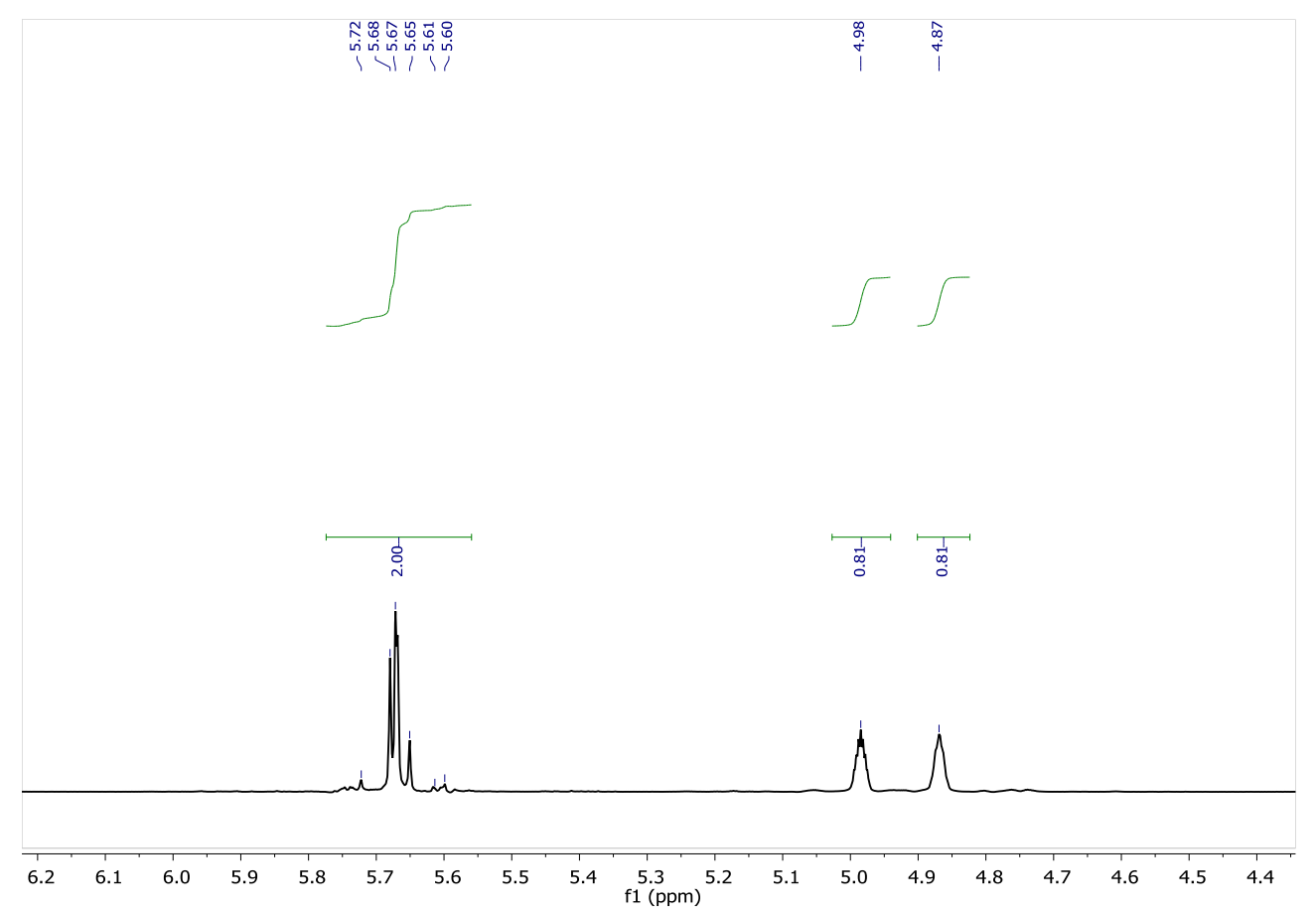

Figure ES3 - Expanded ${ }^{1} \mathrm{H}$ NMR spectrum recorded after 420 s photolysis of 6 (at $1 \mathrm{M}$ substrate concentration) showing the formation of diagnostic alkene peaks of $\mathbf{9}$ and $\mathbf{1 0}$ (Table 2 , entry 10 ). 


\section{Film thickness and mixing measurements}

For these experiments a specific volume of ethanol containing rose bengal (at a known concentration and determined extinction coefficient) was pumped into the flask of the reactor. Estimates of the thickness of the film formed when the flask rotated were obtained spectroscopically using a $6 \mathrm{~V} / 0.6 \mathrm{~W}$ incandescent bulb (similar to that is in an electric torch) attached onto a long rod that was passed down the centre and into the reaction flask, as shown in Figure ES5. Absorption spectra were collected whilst the flask is rotating using a fixed position fibre optic cable (P-400-1-UV-VIS) connected to an Ocean optics USB2000+ spectrometer; Figure ES5 shows some selected spectra.

We found that the behaviour of fluid falls into three types, namely a thin film (F), a puddle (P) and a thicker band (B) as illustrated in Figure ES6. These types were present either in isolation or as a combination dependent on the flask size, solution volume and rotation speed. For each rotation speed measured, 20 individual spectra were collected-with each spectrum comprising of a minimum of 20 scans. The average absorbance value determined for each set of spectra was converted to film-thickness using the Beer-Lambert law and the results are detailed in Table ES1.

For the mixing experiments, a similar procedure was followed. Following the pumping in and film thickness measurement of the initial dye (rose bengal) a separate pump was then used to pump in the second dye (methylene blue). Methylene blue was chosen due to its contrasting absorption spectrum. The rotation speeds investigated were 75, 100, 125 and 150 RPM for the mixing of addition of $2.5 \mathrm{~mL}$ methylene blue in EtOH solution mixing with $7.5 \mathrm{~mL}$ of rose Bengal in EtOH solution. Absorption spectra were recorded of the mixing of the thin film (every $400 \mathrm{~ms}$ ) and recorded until no further changes were apparent in the spectra; example spectra are shown in Figure ES7. The initial growth of the $655 \mathrm{~nm}$ band of methylene blue for each rotation speed was fitted to an either a mono- or bi-exponential. The kinetics indicate that mixing is more efficient at the lower spin speeds when both a thin film and puddle are present. Work is underway to investigate this further. 


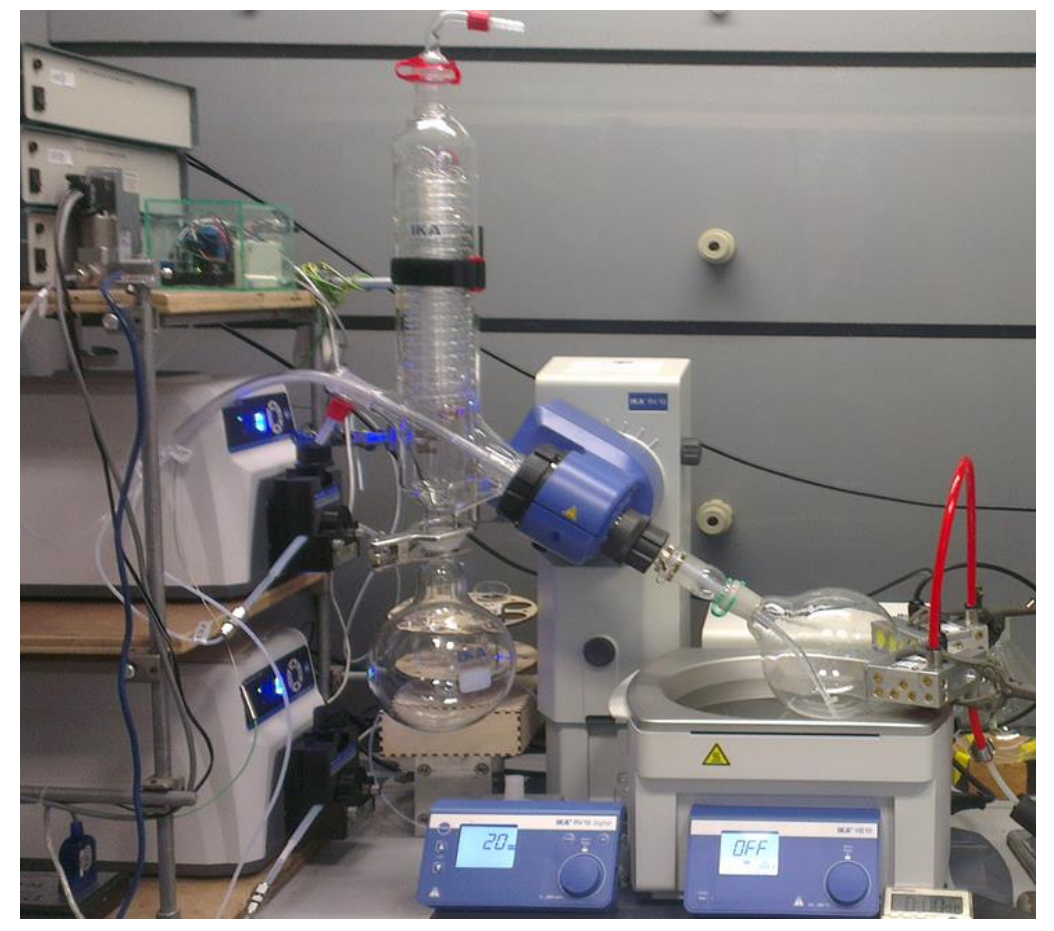

Figure ES4 - Photograph showing photoreactor setup.
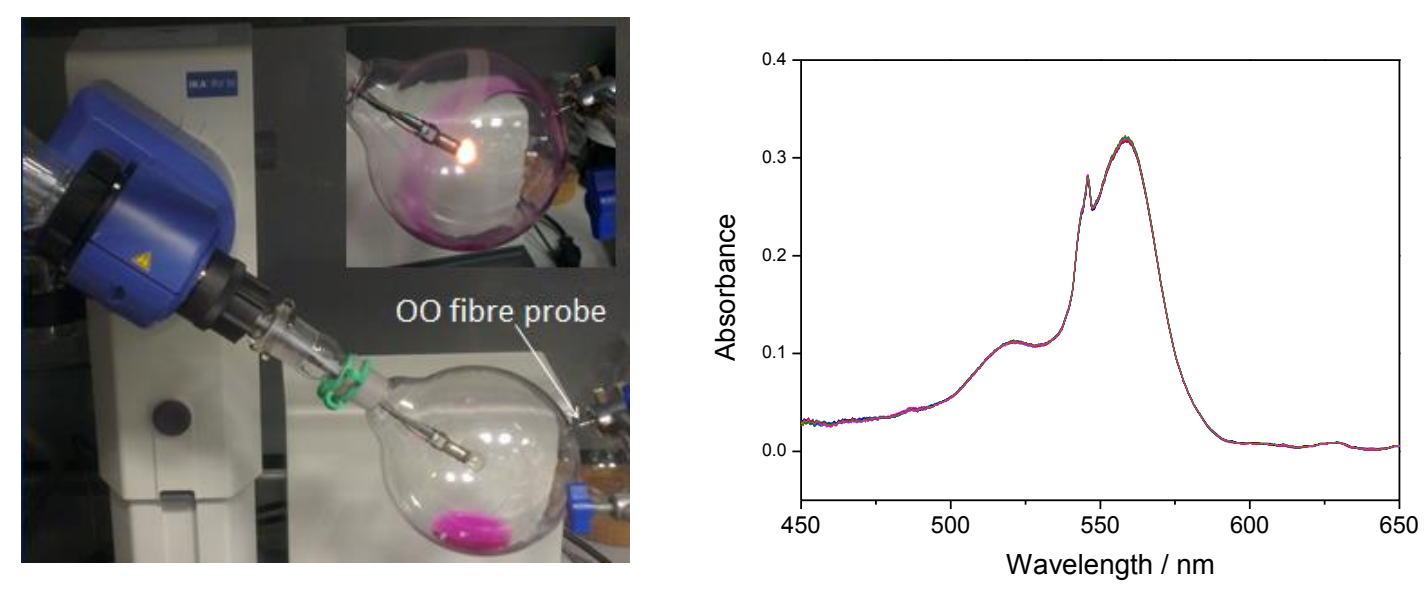

Figure ES5 - Left: Photograph showing setup used for estimating the film thickness using absorption spectroscopy. Inset shows the thin-film with band formed upon 225 RPM rotation of a $1 \mathrm{~L}$ flask containing $5 \mathrm{~mL}$ of rose bengal in EtOH solution. Right: Absorption spectra recorded of $50 \mathrm{~mL}$ of rose bengal in EtOH solution whilst rotating at $200 \mathrm{RPM}$ in a $3 \mathrm{~L}$ flask, 20 overlaid spectra are shown. 


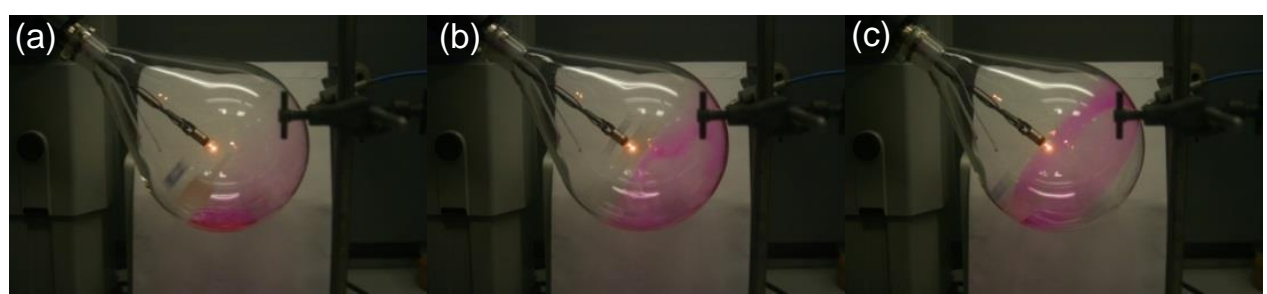

Figure ES6 - Photographs taken during film thickness measurements in a 2 L pear-shaped flask showing the three types of fluid behaviour (a) $15 \mathrm{~mL}$ of solution rotated at $50 \mathrm{RPM}$, assigned as a Film and puddle (F+P), (b) $10 \mathrm{~mL}$ of solution rotated at $150 \mathrm{RPM}$, assigned as a Film (F) and (c) $10 \mathrm{~mL}$ of solution rotated at $200 \mathrm{RPM}$, assigned as a band (B). 
Table ES1 - Estimates of Film thicknesses determined by absorption spectroscopy for a range of volumes, flask sizes and rotation speeds. The probe position was chosen to be a close as possible to where the LEDs were placed in a photolysis experiment. Distance from base-of neck of flask to probe - 1 L RB (12 cm), 1 L PS $(15.5 \mathrm{~cm}), 2$ L PS $(20 \mathrm{~cm}), 3$ L PS $(22 \mathrm{~cm})$.

\begin{tabular}{|c|c|c|c|}
\hline $\begin{array}{c}\text { Rotation } \\
\text { Speed / } \\
\text { RPM }\end{array}$ & Volume / mL & $\begin{array}{c}\text { Film (F) / Puddle } \\
\text { (P) / Band (B) }\end{array}$ & Thickness / $\mu \mathrm{m}$ \\
\hline \multicolumn{4}{|c|}{1 L Round Bottomed (RB) Flask } \\
\hline 50 & 5 & $\mathrm{~F}+\mathrm{P}$ & $60( \pm 10)$ \\
\hline 75 & 5 & $\mathrm{~F}+\mathrm{P}$ & $80( \pm 10)$ \\
\hline 100 & 5 & $\mathrm{~F}+\mathrm{P}$ & $100( \pm 15)$ \\
\hline 125 & 5 & $\mathrm{~F}+\mathrm{P}$ & $130( \pm 20)$ \\
\hline 150 & 5 & $\mathrm{~F}$ & $160( \pm 20)$ \\
\hline 175 & 5 & $\mathrm{~F}$ & $260( \pm 40)$ \\
\hline 200 & 5 & $\mathrm{~B}$ & $540( \pm 70)$ \\
\hline 50 & 10 & $\mathrm{~F}+\mathrm{P}$ & $70( \pm 10)$ \\
\hline 75 & 10 & $\mathrm{~F}+\mathrm{P}$ & $100( \pm 15)$ \\
\hline 100 & 10 & $\mathrm{~F}+\mathrm{P}$ & $125( \pm 15)$ \\
\hline 125 & 10 & $\mathrm{~F}+\mathrm{P}$ & $157( \pm 20)$ \\
\hline 150 & 10 & $\mathrm{~F}+\mathrm{P}$ & $192( \pm 30)$ \\
\hline 175 & 10 & $\mathrm{~F}+\mathrm{P}$ & $231( \pm 30)$ \\
\hline 200 & 10 & $\mathrm{~B}$ & $424( \pm 60)$ \\
\hline 225 & 10 & $\mathrm{~B}$ & $1019( \pm 130)$ \\
\hline \multicolumn{4}{|c|}{1 L Pear-Shaped (PS) Flask } \\
\hline 50 & 5 & $\mathrm{~F}+\mathrm{P}$ & $70( \pm 10)$ \\
\hline 75 & 5 & $\mathrm{~F}+\mathrm{P}$ & $90( \pm 15)$ \\
\hline 100 & 5 & $\mathrm{~F}+\mathrm{P}$ & $90( \pm 15)$ \\
\hline 125 & 5 & $\mathrm{~F}+\mathrm{P}$ & $120( \pm 15)$ \\
\hline 150 & 5 & $\mathrm{~F}$ & $160( \pm 20)$ \\
\hline
\end{tabular}




\begin{tabular}{|cccc|}
175 & 5 & $\mathrm{~F}$ & $190( \pm 30)$ \\
200 & 5 & $\mathrm{~F}+\mathrm{B}$ & $430( \pm 60)$ \\
& & & \\
50 & 10 & $\mathrm{~F}+\mathrm{P}$ & $60( \pm 10)$ \\
75 & 10 & $\mathrm{~F}+\mathrm{P}$ & $90( \pm 15)$ \\
100 & 10 & $\mathrm{~F}+\mathrm{P}$ & $120( \pm 15)$ \\
125 & 10 & $\mathrm{~F}+\mathrm{P}$ & $150( \pm 20)$ \\
150 & 10 & $\mathrm{~F}+\mathrm{P}$ & $170( \pm 20)$ \\
175 & 10 & $\mathrm{~F}+\mathrm{P}$ & $200( \pm 30)$ \\
200 & 10 & $\mathrm{~F}+\mathrm{B}$ & $350( \pm 50)$ \\
225 & 10 & $\mathrm{~F}+\mathrm{B}$ & $630( \pm 80)$ \\
\hline
\end{tabular}

\begin{tabular}{|c|c|c|c|}
\hline $\begin{array}{c}\text { Rotation } \\
\text { Speed / } \\
\text { RPM }\end{array}$ & Volume / mL & $\begin{array}{c}\text { Film (F) / Puddle } \\
\text { (P) / Band (B) }\end{array}$ & Thickness / $\mu \mathrm{m}$ \\
\hline \multicolumn{4}{|c|}{2 L Pear-Shaped (PS) Flask } \\
\hline 50 & 5 & $\mathrm{~F}+\mathrm{P}$ & $50( \pm 10)$ \\
\hline 75 & 5 & $\mathrm{~F}+\mathrm{P}$ & $70( \pm 10)$ \\
\hline 100 & 5 & $\mathrm{~F}+\mathrm{P}$ & $80( \pm 10)$ \\
\hline 125 & 5 & $\mathrm{~F}$ & $170( \pm 25)$ \\
\hline 150 & 5 & $\mathrm{~F}+\mathrm{B}$ & $220( \pm 30)$ \\
\hline 175 & 5 & $\mathrm{~B}$ & $440( \pm 55)$ \\
\hline 50 & 10 & $\mathrm{~F}+\mathrm{P}$ & $70( \pm 10)$ \\
\hline 75 & 10 & $\mathrm{~F}+\mathrm{P}$ & $100( \pm 15)$ \\
\hline 100 & 10 & $\mathrm{~F}+\mathrm{P}$ & $130( \pm 20)$ \\
\hline 125 & 10 & $\mathrm{~F}+\mathrm{P}$ & $190( \pm 25)$ \\
\hline 150 & 10 & $\mathrm{~F}$ & $210( \pm 30)$ \\
\hline 175 & 10 & $\mathrm{~B}$ & $480( \pm 60)$ \\
\hline 200 & 10 & $\mathrm{~B}$ & $690( \pm 90)$ \\
\hline
\end{tabular}




\begin{tabular}{cccc}
50 & 15 & $\mathrm{~F}+\mathrm{P}$ & $100( \pm 15)$ \\
75 & 15 & $\mathrm{~F}+\mathrm{P}$ & $140( \pm 20)$ \\
100 & 15 & $\mathrm{~F}+\mathrm{P}$ & $180( \pm 25)$ \\
125 & 15 & $\mathrm{~F}+\mathrm{P}$ & $230( \pm 30)$ \\
150 & 15 & $\mathrm{~F}$ & $250( \pm 30)$ \\
175 & 15 & $\mathrm{~F}+\mathrm{B}$ & $400( \pm 50)$ \\
200 & 15 & $\mathrm{~B}$ & $790( \pm 100)$ \\
& & & \\
50 & 20 & $\mathrm{~F}+\mathrm{P}$ & $90( \pm 15)$ \\
75 & $\mathrm{~F}+\mathrm{P}$ & $120( \pm 15)$ \\
100 & 20 & $\mathrm{~F}+\mathrm{P}$ & $160( \pm 20)$ \\
125 & 20 & $\mathrm{~F}+\mathrm{P}$ & $210( \pm 30)$ \\
150 & 20 & $\mathrm{~F}+\mathrm{P}$ & $250( \pm 30)$ \\
175 & 20 & $\mathrm{~F}$ & $360( \pm 45)$ \\
200 & 20 & $\mathrm{~F}+\mathrm{B}$ & $650( \pm 80)$ \\
225 & 20 & $\mathrm{~B}$ & $1550( \pm 200)$ \\
\hline
\end{tabular}

\begin{tabular}{|cccc|}
\hline $\begin{array}{c}\text { Rotation } \\
\text { Speed / } \\
\text { RPM }\end{array}$ & Volume / mL & $\begin{array}{c}\text { Film (F) / Puddle } \\
\text { (P) / Band (B) }\end{array}$ & Thickness / $\mu$ m \\
& & & \\
\hline \multicolumn{4}{c|}{ 3 L Pear-Shaped (PS) Flask } \\
\hline 50 & 5 & F & $80( \pm 10)$ \\
75 & 5 & F & $90( \pm 10)$ \\
100 & 5 & F & $70( \pm 10)$ \\
125 & 5 & F + B & $210( \pm 30)$ \\
150 & 5 & B & $440( \pm 60)$ \\
75 & 10 & & $110( \pm 20)$ \\
& 10 & F + P & $140( \pm 20)$
\end{tabular}




\begin{tabular}{|c|c|c|c|}
\hline 100 & 10 & $\mathrm{~F}+\mathrm{P}$ & $180( \pm 25)$ \\
\hline 125 & 10 & $\mathrm{~F}$ & $230( \pm 30)$ \\
\hline 150 & 10 & $\mathrm{~F}+\mathrm{B}$ & $330( \pm 40)$ \\
\hline 175 & 10 & B & $660( \pm 80)$ \\
\hline 50 & 20 & $\mathrm{~F}+\mathrm{P}$ & $130( \pm 20)$ \\
\hline 75 & 20 & $\mathrm{~F}+\mathrm{P}$ & $160( \pm 20)$ \\
\hline 100 & 20 & $\mathrm{~F}+\mathrm{P}$ & $210( \pm 30)$ \\
\hline 125 & 20 & $\mathrm{~F}+\mathrm{P}$ & $250( \pm 30)$ \\
\hline 150 & 20 & $\mathrm{~F}+\mathrm{P}$ & $290( \pm 40)$ \\
\hline 175 & 20 & $\mathrm{~F}+\mathrm{B}$ & $580( \pm 70)$ \\
\hline 200 & 20 & $\mathrm{~B}$ & $1300( \pm 160)$ \\
\hline 50 & 30 & $\mathrm{~F}+\mathrm{P}$ & $120( \pm 15)$ \\
\hline 75 & 30 & $\mathrm{~F}+\mathrm{P}$ & $150( \pm 20)$ \\
\hline 100 & 30 & $\mathrm{~F}+\mathrm{P}$ & $200( \pm 30)$ \\
\hline 125 & 30 & $\mathrm{~F}+\mathrm{P}$ & $230( \pm 30)$ \\
\hline 150 & 30 & $\mathrm{~F}+\mathrm{P}$ & $270( \pm 40)$ \\
\hline 175 & 30 & $\mathrm{~F}+\mathrm{B}$ & $400( \pm 50)$ \\
\hline 200 & 30 & $\mathrm{~B}$ & $820( \pm 110)$ \\
\hline 50 & 50 & $\mathrm{~F}+\mathrm{P}$ & $140( \pm 20)$ \\
\hline 75 & 50 & $\mathrm{~F}+\mathrm{P}$ & $180( \pm 20)$ \\
\hline 100 & 50 & $\mathrm{~F}+\mathrm{P}$ & $210( \pm 30)$ \\
\hline 125 & 50 & $\mathrm{~F}+\mathrm{P}$ & $240( \pm 30)$ \\
\hline 150 & 50 & $\mathrm{~F}+\mathrm{P}$ & $280( \pm 40)$ \\
\hline 175 & 50 & $\mathrm{~F}+\mathrm{B}$ & $340( \pm 45)$ \\
\hline 200 & 50 & $\mathrm{~F}+\mathrm{B}$ & $550( \pm 70)$ \\
\hline 225 & 50 & $\mathrm{~B}$ & $1920( \pm 540)$ \\
\hline
\end{tabular}




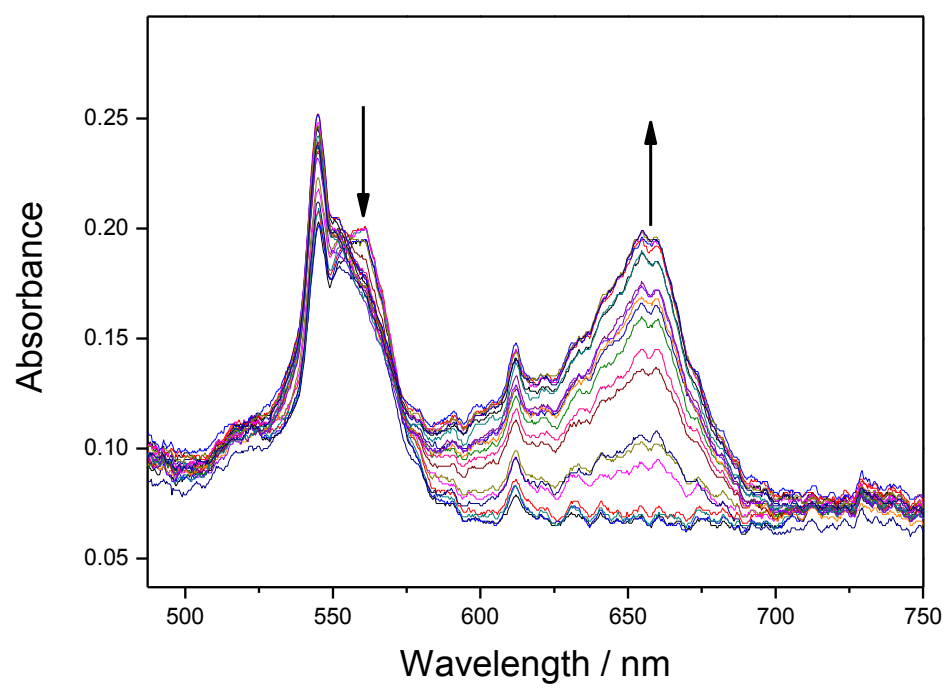

.Figure ES7- Absorption spectra measured of the in-situ mixing in a $3 \mathrm{~L}$ flask (rotating at $100 \mathrm{RPM})$ of EtOH solutions of rose bengal $(7.5 \mathrm{~mL})$ and methylene blue $(2.5 \mathrm{~mL})$. Spectra recorded every $400 \mathrm{~ms}$ using a similar setup to that described above.

\section{Internal temperature measurements and thermal imaging photography}

Internal temperature measurements were obtained by inserting a thermocouple into the reactor and positioning it so that it touched the bottom inside surface of the flask, without moving during rotation. The change in temperature of an $\mathrm{EtOH}$ solution containing $5 \mathrm{mM}$ of rose bengal upon irradiation (with 2 banks of 8000 lumen LEDs) at several flask rotation speeds was logged using a PicoLog data logger and the time needed for the temperature to equilibrate was found to depend on flask rotation speed. At lower rotation speeds the equilibrium time required was found to be longer that those measured at higher rotation speeds. The overall increase in temperature during photolysis was also found to be smaller at higher rotation speeds, due to the removal of heat using the water bath of the reactor. Thermal imaging photography (FLIR-T400 camera with FLIR Quick report software) was used to monitor the external temperature of the reactor flask at several rotation speeds, whilst spinning, both in the dark and during irradiation. Selected photographs are shown in Figure ES8. In the dark, it was observed that as the rotation speed increases, the temperature of the external thin film of water (from the bath) becomes more uniform across the flask. Upon irradiation, the temperature of this external film is also seen to be uniform. Both of these observations indicate that the rotation of the flask is important not only for generating the internal reaction thin-film but in maintaining a consistent reaction temperature using the supplied standard water bath. 

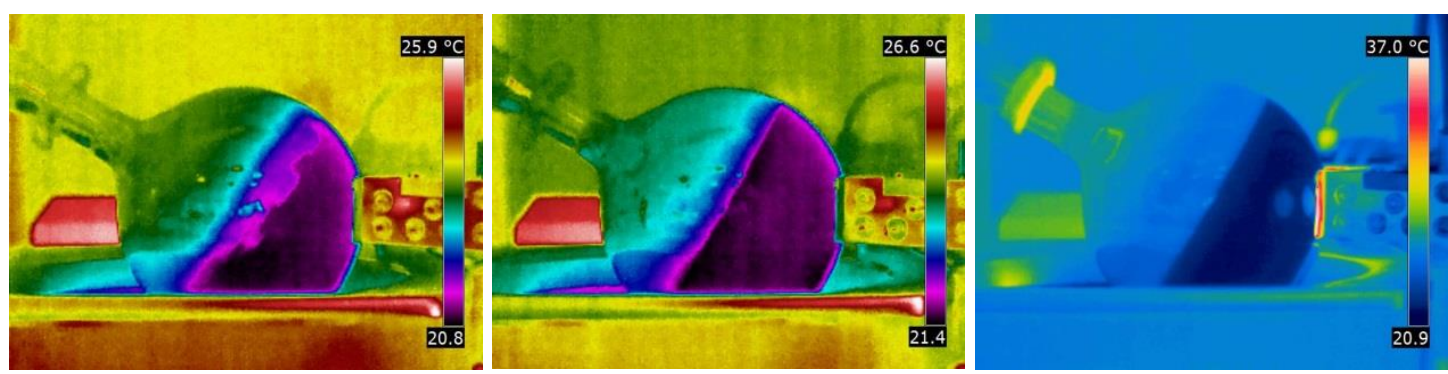

Figure ES8 Thermal imaging photographs of a 1 L RB flask rotating at specific speeds whilst in a water bath at room temperature. Left in the dark, an empty flask rotating at 10 RPM - the cool film of water is clearly visible on the outside of the flask. Centre an empty flask in the dark, rotating at 175 RPM. Right during irradiation of a filled flask with 2 banks of high power LEDs, containing $20 \mathrm{~mL}$ EtOH/rose bengal solution, rotating at $200 \mathrm{RPM}$. 